\title{
フィードバック指数受動性に基づく多入出力非線形系に対する適応出力 フィードバック制御
}

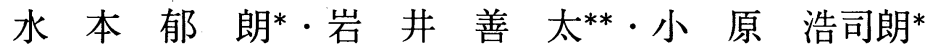

\begin{abstract}
Adaptive Output Feedback Control for MIMO Nonlinear Systems based on Feedback Exponential Passivity
\end{abstract}

Ikuro Mizumoto*, Zenta IwaI** and Koshiro Kohara*

\begin{abstract}
The property of systems that can be rendered exponentially passive via output feedback is called output feedback exponential passivity. The design of adaptive output feedback control for output feedback exponentially passive MIMO nonlinear systems is proposed. Further, the alleviation of the output feedback exponentially passive conditions by introducing a parallel feedforward compensator (PFC) will be considered and design principle for the PFC is derived. The new concrete design scheme for the PFC is also given.
\end{abstract}

Key Words: adaptive control, nonlinear systems, MIMO systems, feedback exponential passivity, parallel feed forward compensator

\section{1. 緒言}

システムの受動性 (Passivity) は, 非線形系の安定性を解 析する重要なツールとして知られている。この受動性の概念 を用いることにより，たとえば，受動的なシステムは，単純 な定ゲイン出力フィードバックにより漸近安定とすることが できる1)ことや, また, 強受動的 (strictly passive) なシステ ムと受動的なシステムとの負のフィードバック結合は, 安定

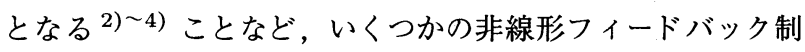
御系の設計において注目すべき結果が得られている．後者の 結果は, 安定な適応制御系を設計する上での重要な結果の 1 つで，受動定理として知られている．ただ，残念なことに， この非線形系の受動性は, ほとんどの実システムにとって満 足されない条件となっている。この点に対し, Byrnesら は, 状態フィードバックによりシステムが (強) 受動化可能 な, すなわちフィードバック (強) 受動 (feedback (strictly) passive: $\mathrm{F}(\mathrm{S}) \mathrm{P})$ なための条件が，1) システムの相対次数が $\{1,1, \cdots, 1\}$ であり，2) システムが弱最小位相 (最小位相) (weakly minimum phase(minimum phase)), であることを 示し, さらに Seron $ら^{5)}$ は, 上述の feedback passive (FP)

* 熊本大学大学院自然科学研究科 熊本市黒髪 2-39-1

** 熊本大学工学部 熊本市黒髮 2-39-1

* Graduate School of Science and Technology, Kumamoto University, Kumamoto

** Faculty of Engineering, Kumamoto University, Kumamoto

(Received March 31, 2000)

(Revised December 6, 2000)
条件のもとで, 適応制御系の設計法の提案を行っている.し かしながら，上述の FP 条件もまた，実システムにとっては 厳しい制約であり，また，たとえシステムが FPであっても， 制御系を受動化するためには，すべての状態変数の情報が 必要とされていた。この問題に対する 1 つの解として近年 Backstepping 法に基づく制御系設計法が提案されている ${ }^{6)}$. しかしこの手法は, 基本的には制御系を構成するためにはシ ステムの次数に関する情報が必要であり，高次のシステムに 対しては, その構造はかなり複雑となる.

さて, 線形系においてもこの FP 性はこれまで意欲的に 議論されており, 上述した FP 条件は, 線形系では, almost strictly positive real (ASPR) 条件として知られている7). この線形系に対する ASPR 条件は, 状態フィードバックに よる (強) 受動化だけでなく同時に出力フィードバックによ るシステムの (強) 受動化をも実現することができる，この ことから, 線形系においては, 高次のシステムに対しても プラントの ASPR 性に基づいてプラントの次数に依存しな い出力フィードバック形式の構造の簡単な適応制御系が設計 できることが知られている ${ }^{8)}$. しかし, 非線形系の場合と 同様に線形系においてもこの条件は厳しい制約条件となっ ていたこの点に関して, 線形系においては, 1 つの解決策 として, 並列フィードフォワード補償 (parallel feedforward compensator: PFC) の導入が検討されている ${ }^{9) 〜 12)}$ 。この 手法は, PFC を付加した拡張系を ASPRにすることで, こ の拡張系に対し, システムの ASPR 性に基づく制御手法が 適用できるというものであり，そのような PFCの具体的な 
設計法もすでにいくつか提案されている10) 12). さらに，近 年, 1 入出力非線形系に対する適応制御系設計法に関して, PFC 導入の概念がすでに一部応用されている ${ }^{13) \sim 15) . ~ し か ~}$ し，現在までのところ多入出力系に対する十分な検討はなさ れていない。

本報告では, 文献 16)で考察されている出カフィードバック 指数受動性 (output feedback exponential passivity:OFEP) のもと多入出力非線形系に対して, 出力フィードバック形式 のシステムの次数に依存しない適応制御系が簡単に構成でき ることを示す，さらに，対象システムが OFEP 条件を満足し ていない場合に対し，この OFEP 条件を緩和するための非 線形系に対する具体的な PFC 設計法の提案を行う.

\section{2. 準備（強受動性とシステム表現）}

つぎのように表されるAffine 非線形システムを考える.

$$
\begin{aligned}
\dot{\boldsymbol{x}}(t) & =\boldsymbol{f}(\boldsymbol{x})+G(\boldsymbol{x}) \boldsymbol{u}(t) \\
\boldsymbol{y}(t) & =\boldsymbol{h}(\boldsymbol{x})
\end{aligned}
$$

ここに, $\boldsymbol{x}(t) \in R^{n}$ は状態べクトル, $\boldsymbol{u}(t) \in R^{m}$ は入 力, $y(t) \in R^{m}$ は出力である.また, $\boldsymbol{f}(\boldsymbol{x}): R^{n} \rightarrow R^{n}$, $G(\boldsymbol{x})=\left[\boldsymbol{g}_{1}, \boldsymbol{g}_{2}, \cdots, \boldsymbol{g}_{m}\right]: R^{n} \rightarrow R^{n \times m}$ および $\boldsymbol{h}(\boldsymbol{x})=$ $\left[h_{1}, h_{2}, \cdots, h_{m}\right]^{T}: R^{n} \rightarrow R^{m}$ は, 滑らかであり, $\boldsymbol{f}(\mathbf{0})=\mathbf{0}$, $\boldsymbol{h}(\mathbf{0})=\mathbf{0}$ とする.

ここでは, まず準備として（2.1）で表されるシステムの強 受動性および相対次数に関する定義を示しておく.

【定義 1】(強受動性： strict passivity) ${ }^{1), 5)}$ システム $(2.1)$ は任意の $t \geq 0, \boldsymbol{u} \in R^{m}, \boldsymbol{x}(\mathbf{0}) \in R^{n}$ に対し，

$$
V(\boldsymbol{x}(t))-V(\boldsymbol{x}(0)) \leq \int_{0}^{t} \boldsymbol{y}(\tau)^{T} \boldsymbol{u}(\tau) d \tau-\int_{0}^{t} \zeta(\boldsymbol{x}(\tau)) d \tau
$$

を満足するような非負の連続関数 $V: R^{n} \rightarrow R, V(0)=0$, $\forall t \geq 0$ および正定値関数 $\zeta: R^{n} \rightarrow R$ 孝在するとき, 強受 動的 (strictly passive) であるという.

【定義 2】(指数受動性：exponential passivity) ${ }^{16)}$ 強受動 的なシステム $(2.1)$ は， $(2.1)$ の任意の解 $\boldsymbol{x}(t)$ に対し，

$$
\begin{aligned}
& \alpha_{1}\|x\|^{2} \leq V(x) \leq \alpha_{2}\|x\|^{2} \\
& \alpha_{3}\|x\|^{2} \leq \zeta(x)
\end{aligned}
$$

なる関係を満足する正定数 $\alpha_{1}, \alpha_{2}, \alpha_{3}$ が存在するとき, 指数 受動的 (exponentially passive) であるという.

【定義 3】(相対次数： relative degree) ${ }^{17)}$ システム (2.1) は, すべての $\boldsymbol{x} \in R^{n}$ に対し, すべての $i=1, \cdots, m$, $j=1, \cdots, m, k=1, \cdots, \gamma_{i}-2$ で

$$
L_{g_{j}} L_{f}^{k} h_{i}(x)=0
$$

であり,さらに $m \times m$ 行列

$$
\widetilde{B}(\boldsymbol{x})=\left[\begin{array}{ccc}
L_{g_{1}} L_{f}^{\gamma_{1}-1} h_{1}(\boldsymbol{x}) & \cdots & L_{g_{m}} L_{f}^{\gamma_{1}-1} h(\boldsymbol{x}) \\
L_{g_{1}} L_{f}^{\gamma_{2}-1} h_{2}(\boldsymbol{x}) & \cdots & L_{g_{m}} L_{f}^{\gamma_{2}-1} h_{2}(\boldsymbol{x}) \\
\vdots & & \vdots \\
L_{g_{1}} L_{f}^{\gamma_{m}-1} h_{m}(\boldsymbol{x}) & \cdots & L_{g_{m}} L_{f}^{\gamma_{m}-1} h_{m}(\boldsymbol{x})
\end{array}\right]
$$

がすべての $\boldsymbol{x} \in R^{n}$ で正則であるとき, (一様) 相対次数 $\left\{\gamma_{1}, \gamma_{2}, \cdots, \gamma_{m}\right\}$ を持つという。ここに, $L_{f} h_{i}, L_{g_{j}} h_{i}$ はそ れぞれ $h_{i}(\boldsymbol{x})$ の $\boldsymbol{f}(\boldsymbol{x}), \boldsymbol{g}_{i}(\boldsymbol{x})$ によるリー $(\mathrm{Lie})$ 微分である.

さて, システム $(2.1)$ が相対次数 $\left\{\gamma_{1}, \cdots, \gamma_{m}\right\}$ を持ち, さ らに以下の仮定が満足されているとする.

\section{[仮定 1]}

$$
\widetilde{G}(\boldsymbol{x})=\left[\widetilde{\boldsymbol{g}}_{1}(\boldsymbol{x}), \cdots, \widetilde{\boldsymbol{g}}_{m}(\boldsymbol{x})\right]=G(\boldsymbol{x}) \widetilde{B}(\boldsymbol{x})^{-1}
$$
とおくとき，

（1） ベクトル場 $\widetilde{\boldsymbol{g}}_{1}, \cdots, \widetilde{\boldsymbol{g}}_{m}$ は完備 (complete)

(2) ベクトル場 $\widetilde{\boldsymbol{g}}_{1}, \cdots, \widetilde{\boldsymbol{g}}_{m}$ は可換 (commute)

((2)は, $\operatorname{span}\left\{\boldsymbol{g}_{1}, \cdots, \boldsymbol{g}_{m}\right\}$ がインボリューティブと同じ条件)

このとき, ある滑らかな正則変換 $z=\left[z_{1}, \cdots, z_{n}\right]^{T}=\Phi(\boldsymbol{x})$ が存在し, システム $(2.1)$ が,

$$
\begin{aligned}
\dot{\boldsymbol{z}}_{\xi}(t) & =A \boldsymbol{z}_{\xi}(t)+B \widetilde{\boldsymbol{a}}\left(\boldsymbol{z}_{\xi}, \boldsymbol{z}_{q}\right)+B \widetilde{B}\left(\boldsymbol{z}_{\xi}, \boldsymbol{z}_{q}\right) \boldsymbol{u}(t) \\
\dot{\boldsymbol{z}}_{q}(t) & =\boldsymbol{q}\left(\boldsymbol{z}_{\xi}, \boldsymbol{z}_{q}\right) \\
\boldsymbol{y}(t) & =C \boldsymbol{z}_{\xi}(t)
\end{aligned}
$$

と表されることが知られている17),18).ここに，

$$
\begin{aligned}
& \boldsymbol{z}_{\xi}(t)=\left[z_{1}(t), \cdots, z_{\gamma}(t)\right]^{T}, \quad \gamma=\sum_{i=1}^{m} \gamma_{i} \\
& \boldsymbol{z}_{q}(t)=\left[z_{\gamma+1}(t), \cdots, z_{n}(t)\right]^{T} \\
& \widetilde{\boldsymbol{a}}(\mathbf{0}, \mathbf{0})=\left[L_{f}^{\gamma_{1}} h_{1}(\mathbf{0}), \cdots, L_{f}^{\gamma_{m}} h_{m}(\mathbf{0})\right]^{T}=\mathbf{0} \\
& \boldsymbol{q}(\mathbf{0}, \mathbf{0})=\mathbf{0}
\end{aligned}
$$

であり，

$$
\begin{aligned}
& A=\operatorname{diag}\left[A_{i}\right]_{i=1, \cdots, m}, B=\operatorname{diag}\left[\boldsymbol{b}_{i}\right]_{i=1, \cdots, m} \\
& C=\operatorname{diag}\left[\boldsymbol{c}_{\boldsymbol{i}}^{T}\right]_{i=1, \cdots, m}
\end{aligned}
$$

$$
\begin{aligned}
A_{i} & =\left[\begin{array}{ccccc}
0 & 1 & 0 & \cdots & 0 \\
0 & 0 & 1 & \cdots & 0 \\
\cdot & \cdot & . & \cdots & \cdot \\
0 & 0 & 0 & \cdots & 1 \\
0 & 0 & 0 & \cdots & 0
\end{array}\right], \boldsymbol{b}_{i}=\left[\begin{array}{c}
0 \\
\vdots \\
0 \\
1
\end{array}\right] \\
\boldsymbol{c}_{i}^{T} & =\left[\begin{array}{llll}
1 & 0 & \cdots & 0
\end{array}\right]
\end{aligned}
$$

$A_{i} \in R^{\gamma_{i} \times \gamma_{i}}, \boldsymbol{b}_{i}, \boldsymbol{c}_{i} \in R^{\gamma_{i}}$

である.(2.8)のシステム表現は, Isidori の標準形と呼ばれ ている．以下，(2.1)に対する $(2.8)$ のシステム表現を単に標 準形と呼ぶことにする.

本報告では, 以下, (2.8) の標準形に変換可能なシステム (2.1) を対象に考察を行う. 


\section{3. 出カフィードバック指数受動性に基づく適応制御}

ある状態フィードバックにより構成された閉ループ系が受 動化可能な場合, そのシステムはフィードバック受動 (FP) なシステムと呼ばれる5)。ここでは, 出力フィードバックに より指数受動化可能な出力フィードバック指数受動 (OFEP) なシステムに対する適応制御手法の提案を行う.

【定義 4】(出カフィードバック指数受動：output feedback exponentially passive(OFEP)) システム (2.1) は, 構成 された閉ループ系が $\boldsymbol{v}(t)$ から $\boldsymbol{y}(t)$ に関して指数受動的となる 滑らかな出力フィードバック：

$$
\begin{array}{r}
\boldsymbol{u}(t)=\boldsymbol{r}(\boldsymbol{y})+\Omega(\boldsymbol{y}) \boldsymbol{v}(t) \\
\boldsymbol{r}(\mathbf{0})=\mathbf{0}, \quad \Omega(\mathbf{0})=0
\end{array}
$$

が存在するとき, 出カフィードバック指数受動 (OFEP) であ ると呼ばれる。

標準形 (2.8) を持つシステム (2.1) が OFEP であるための 十分条件は，すでにつぎのような条件が明らかにされてい る $^{16)}$.

[出カフィードバック指数受動条件 (OFEP 条件)]

(1) システム (2.1) は, 相対次数 $\{1, \cdots, 1\}$ を持つ.

(2) システム (2.1) は, 指数最小位相である.すなわち, 標準形 $(2.8)$ により定義されるゼロダイナミクス：

$$
\dot{\boldsymbol{z}}_{q}(t)=\boldsymbol{q}\left(0, \boldsymbol{z}_{q}\right)
$$

は, 指数安定である.

(3) 標準形の $\widetilde{\boldsymbol{a}}\left(\boldsymbol{z}_{\xi}, \boldsymbol{z}_{q}\right)$ および $\boldsymbol{q}\left(\boldsymbol{z}_{\xi}, \boldsymbol{z}_{q}\right)$ は, $\left(\boldsymbol{z}_{\xi}, \boldsymbol{z}_{q}\right)$ に 関して Lipschitzである。

(4) 標準形の $\widetilde{B}\left(\boldsymbol{z}_{\xi}, \boldsymbol{z}_{q}\right)$ は, $\widetilde{B}_{0} \widetilde{B}_{1}(\boldsymbol{y}), \widetilde{B}_{0}=\widetilde{B}_{0}^{T}>0$ と 表される。

上述の条件は,

$$
\boldsymbol{u}(t)=-\widetilde{B}_{1}(\boldsymbol{y})^{-1} K \boldsymbol{y}(t)+\widetilde{B}_{1}(\boldsymbol{y})^{-1} \boldsymbol{v}(t)
$$

なる出力フィードバックにより構成されたシステム $(2.1)$ に 対する閉ループ系が $K^{T}+K \geq K_{0}$ なるすべての $K$ に対し， $\boldsymbol{v}(t)$ から $\boldsymbol{y}(t)$ に関して指数受動的となる正定対称行列 $K_{0}$ が 存在するという意味で, 出力フィードバック指数受動条件を 与えている (付録 A 参照).

いま，上記の OFEP 条件を満足するシステム $(2.1)$ を考え る、ただし，標準形の $\widetilde{B}_{1}(\boldsymbol{y})$ は既知とする。このシステムに 対し, 制御入力を適応的に

$$
\begin{aligned}
\boldsymbol{u}(t) & =-\widetilde{B}_{1}(\boldsymbol{y})^{-1} K(t) \boldsymbol{y}(t) \\
\dot{K}(t) & =\boldsymbol{y}(t) \boldsymbol{y}(t)^{T} \Gamma, \Gamma=\Gamma^{T}>0
\end{aligned}
$$

と構成する.このとき，構成された制御系の安定性に関し， 以下の定理が成立する。
《定理 1》 システム $(2.1)$ は, 標準形 $(2.8)$ に変換可能である とする.このとき，システム (2.1) が OFEP 条件を満足して いるならば, 制御入力を (3.4), (3.5) と構成するとき, 制御 系内の全信号は有界であり, さらに, $\lim _{t \rightarrow \infty} \boldsymbol{y}(t)=0$ となる. (証明)： OFEP 条件を満足するジステムは相対次数 $\{1, \cdots, 1\}$ を持つことから，標準形は

$$
\begin{aligned}
& \dot{\boldsymbol{y}}(t)=\widetilde{\boldsymbol{a}}\left(\boldsymbol{y}, \boldsymbol{z}_{q}\right)+\widetilde{B}(\boldsymbol{y}) \boldsymbol{u}(t) \\
& \dot{\boldsymbol{z}}_{q}(t)=\boldsymbol{q}\left(\boldsymbol{y}, \boldsymbol{z}_{q}\right)
\end{aligned}
$$

と表される.

いま, 制御入力を

$$
\begin{aligned}
\boldsymbol{u}(t) & =-\widetilde{B}_{1}(\boldsymbol{y})^{-1} K^{*} \boldsymbol{y}(t)+\widetilde{B}_{1}(\boldsymbol{y})^{-1} \boldsymbol{v}(t) \\
\boldsymbol{v}(t) & =-\Delta K(t) \boldsymbol{y}(t) \\
\Delta K(t) & =K(t)-K^{*}
\end{aligned}
$$

と書き表す.ここに， $K^{*}$ は, システム $(3.6)$ を指数受動化す る出力フィードバックゲインである. OFEP 条件のもと, こ のような出力フィードバックゲインは必ず存在する（付録 A 参照)。このとき, 構成された閉ループ系は

$$
\begin{aligned}
& \dot{\boldsymbol{y}}(t)=\widetilde{\boldsymbol{a}}\left(\boldsymbol{y}, \boldsymbol{z}_{q}\right)-\widetilde{B}_{0} K^{*} \boldsymbol{y}(t)+\widetilde{B}_{0} \boldsymbol{v}(t) \\
& \dot{\boldsymbol{z}}_{q}(t)=\boldsymbol{q}\left(\boldsymbol{y}, \boldsymbol{z}_{q}\right)
\end{aligned}
$$

と表される。ここで, OFEP 条件 (2) より, ゼロダイナミ クス:

$$
\dot{\boldsymbol{z}}_{q}(t)=\boldsymbol{q}\left(\mathbf{0}, \boldsymbol{z}_{q}\right)
$$

が指数安定であり, さらに, OFEP 条件 (3)より, $\boldsymbol{q}\left(\boldsymbol{y}, \boldsymbol{z}_{q}\right)$ が $\left(\boldsymbol{y}, \boldsymbol{z}_{q}\right)$ に関して Lipschitzであることから，次式を泗足す る正定值関数 $W\left(z_{q}\right)$ が存在する ${ }^{19), 20)}$.

$$
\begin{aligned}
& \frac{\partial W\left(\boldsymbol{z}_{q}\right)}{\partial \boldsymbol{z}_{q}} \boldsymbol{z}_{q}\left(\mathbf{0}, \boldsymbol{z}_{q}\right) \leq-\boldsymbol{\alpha}_{1}\left\|\boldsymbol{z}_{q}(t)\right\|^{2} \\
& \left\|\frac{\partial W\left(\boldsymbol{z}_{q}\right)}{\partial \boldsymbol{z}_{q}}\right\| \leq \alpha_{2}\left\|\boldsymbol{z}_{q}(t)\right\| \\
& \alpha_{3}\left\|\boldsymbol{z}_{q}(t)\right\|^{2} \leq\left\|W\left(\boldsymbol{z}_{q}\right)\right\| \leq \alpha_{4}\left\|\boldsymbol{z}_{q}(t)\right\|^{2}
\end{aligned}
$$

ここに， $\alpha_{1} \sim \alpha_{4}$ は，ある正定数である．このとき，

$$
V_{1}\left(\boldsymbol{y}, \boldsymbol{z}_{q}\right)=W\left(\boldsymbol{z}_{q}\right)+\frac{1}{2} \boldsymbol{y}(t)^{T} \widetilde{B}_{0}^{-1} \boldsymbol{y}(t)
$$

なる正定值関数を考えると，その時間微分が

$$
\dot{V}_{1}\left(\boldsymbol{y}, \boldsymbol{z}_{q}\right) \leq \boldsymbol{y}(t)^{T} \boldsymbol{v}(t)-c_{1}\left\|\boldsymbol{z}_{q}(t)\right\|^{2}-c_{2}\|\boldsymbol{y}(t)\|^{2}(3.14)
$$
と評価できる正定数 $c_{1}, c_{2}$ が存在する (付録 $\mathrm{A}$ 参照). そこで， つぎの正定值関数を考える.

$$
V(t)=V_{1}\left(\boldsymbol{y}, \boldsymbol{z}_{q}\right)+\frac{1}{2} \operatorname{tr}\left[\Delta K(t) \Gamma^{-1} \Delta K(t)^{T}\right]
$$

$V(t)$ の時間微分は, $(3.5),(3.8),(3.14) よ り$,

$$
\begin{aligned}
\dot{V}(t) & =\dot{V}_{1}\left(\boldsymbol{y}, \boldsymbol{z}_{q}\right)+\operatorname{tr}\left[\Delta K(t) \Gamma^{-1} \Delta \dot{K}(t)^{T}\right] \\
& =\dot{V}_{1}\left(\boldsymbol{y}, \boldsymbol{z}_{q}\right)+\operatorname{tr}\left[\Delta K(t) \boldsymbol{y}(t) \boldsymbol{y}(t)^{T}\right] \\
& =\dot{V}_{1}\left(\boldsymbol{y}, \boldsymbol{z}_{q}\right)+\boldsymbol{y}(t)^{T} \Delta K(t) \boldsymbol{y}(t) \\
& =\dot{V}_{1}\left(\boldsymbol{y}, \boldsymbol{z}_{q}\right)-\boldsymbol{y}(t)^{T} \boldsymbol{v}(t) \\
& \leq-c_{1}\left\|\boldsymbol{z}_{q}(t)\right\|^{2}-c_{2}\|\boldsymbol{y}(t)\|^{2} \leq 0
\end{aligned}
$$

と評価できる。すなわち, $\boldsymbol{y}(t), \boldsymbol{z}_{q}(t)$ および $\Delta K(t)$ は有界 である.ここで，当然のことながら $K^{*}$ としては，(A.8) 式 
を満足する有界な定数行列を考えており（厳密には, ここで は $K^{*}$ として, $\lambda_{\min }\left(K^{* T}+K^{*}\right)=\beta_{0}+c_{2}$ となる定数行列 を考えている， $\beta_{0}$ は (A.7) 式で定義される有界な正定数であ る.)，この有界な定数行列 $K^{*}$ を用いて構成された正定値関 数 $V(t)$ を考えていることから, $\Delta K(t)$ の有界性より $K(t)$ も有界であることがわかる。このことから，制御系内の全信 号が有界となることは明らかである，さらに，(3.16)より， $\boldsymbol{y}, z_{q} \in L_{2}$ であることもわかる. また，(3.6)より，明らかに $\dot{\boldsymbol{y}}, \dot{z}_{q} \in L_{\infty}$ である. よって, $\boldsymbol{y}, \boldsymbol{y} \in L_{\infty}, \boldsymbol{y} \in L_{2}$ であること から, $r(t)=\|\boldsymbol{y}(t)\|^{2}$ とおくと, $r(t)$ は一様連続であり, か $\supset \lim _{t \rightarrow \infty} \int_{0}^{t} r(\tau) d \tau$ が存在し有界である.よって, Barbalat の 補題 ${ }^{20)}$ より, $\lim _{t \rightarrow \infty} r(t)=0$, すなわち $\lim _{t \rightarrow \infty} \boldsymbol{y}(t)=0$ が得ら れる。

注意: 提案手法では, オブザーバを必要としないことから， $\widetilde{B}_{1}(\boldsymbol{y})$ が既知であれば制御対象の次数にまったく依存しな い制御系が構成できる.すなわち, 高次のシステムに対して もシンプルな制御器の構造を保つことができ, 次数のミス マッチに対してもロバストな制御系が構成可能である。ただ, $\widetilde{B}_{1}(\boldsymbol{y})$ を得るためには，少なくとも元の非線形プラントの入 力がどのようにシステムに印加されているのかという情報 （相対次数の情報もこれから得られる）と $G(\boldsymbol{x})$ 拉よび $\boldsymbol{h}(\boldsymbol{x})$ がどの状態量に依存しているかという情報, およびその状態 量に関係する微分方程式 $(\boldsymbol{f}(\boldsymbol{x})$ の要素) の構造に関する情 報が必要となる。しかし，他の部分（これらに関係のないこ とが解っている高次の項やそのために無視した部分）の情報 は必要としないことから, 正確な制御対象の次数に関する情 報は必要とはならない。

\section{PFC 導入によるシステムの出カフィードバッ ク指数受動化}

\section{4. $1 \mathrm{PFC}$ の基本的設計条件}

いま, 相対次数 $\left\{\gamma_{1}, \gamma_{2}, \cdots, \gamma_{m}\right\}, \gamma_{i}>1$ を持ち, 標準形 (2.8) で表されるシステム $(2.1)$ を考える.このシステムは, つぎの仮定を满足しているものとする.

\section{[仮定 2]}

(1) システムは指数最小位相である。

(2) 標準形の $\widetilde{\boldsymbol{a}}\left(\boldsymbol{z}_{\xi}, \boldsymbol{z}_{q}\right)$ および $\boldsymbol{q}\left(\boldsymbol{z}_{\xi}, \boldsymbol{z}_{q}\right)$ は, $\left(\boldsymbol{z}_{\xi}, \boldsymbol{z}_{q}\right)$ に 関して Lipschitzである.

(3) 標準形の $\widetilde{B}\left(\boldsymbol{z}_{\xi}, \boldsymbol{z}_{q}\right)$ は, $\widetilde{B}(\boldsymbol{y})$ と表され, 既知である.

上記仮定を満足するシステムに対して，任意の $n_{f}$ 次の並 列フィードフォワード補償 $(\mathrm{PFC})$ をつぎのように構成する.

$$
\begin{aligned}
& \dot{\boldsymbol{z}}_{f}(t)=A_{f} \boldsymbol{z}_{f}(t)+B_{f} \widetilde{B}(\boldsymbol{y}) \boldsymbol{u}(t) \\
& \boldsymbol{y}_{f}(t)=C_{f} \boldsymbol{z}_{f}(t)
\end{aligned}
$$

このとき, $\mathrm{PFC}$ を付加した搪張系は,

$$
\begin{aligned}
& \dot{\boldsymbol{z}}_{a}(t)=A_{a} \boldsymbol{z}_{a}(t)+B_{a} \widetilde{B}(\boldsymbol{y}) \boldsymbol{u}(t)+B_{1} \widetilde{\boldsymbol{a}}\left(\boldsymbol{z}_{\xi}, \boldsymbol{z}_{q}\right) \\
& \dot{\boldsymbol{z}}_{q}(t)=\boldsymbol{q}\left(\boldsymbol{z}_{\xi}, \boldsymbol{z}_{q}\right)
\end{aligned}
$$

$$
\boldsymbol{y}_{a}(t)=\boldsymbol{y}(t)+\boldsymbol{y}_{f}(t)=C_{a} z_{a}(t)
$$

と表される。ここに， $\boldsymbol{z}_{a}(t)=\left[\boldsymbol{z}_{\xi}(t)^{T}, \boldsymbol{z}_{f}(t)^{T}\right]^{T}$ であり，

$$
\begin{aligned}
A_{a} & =\left[\begin{array}{cc}
A & 0 \\
0 & A_{f}
\end{array}\right], B_{a}=\left[\begin{array}{c}
B \\
B_{f}
\end{array}\right] \\
C_{a} & =\left[C, C_{f}\right], B_{1}=\left[\begin{array}{c}
B \\
0
\end{array}\right]
\end{aligned}
$$

である。

ここで, PFCが拡張系の線形部 $\left(A_{a}, B_{a}, C_{a}\right)$ が ASPR，す なわち，1）相対 MacMillan 次数が $m$ （相対次数 $\{1, \cdots, 1\}$, 2) 最小位相，3) 高周波ゲイン行列 $C_{a} B_{a}$ が正定対称行列, と なるように設計されているものと仮定する.

このとき, $\left(A_{a}, B_{a}, C_{a}\right)$ の相対 MacMillan 次数が $m$ であることから，つぎの関係を满足する行列， $N \in$ $R^{\left(\gamma-m+n_{f}\right) \times\left(\gamma+n_{f}\right)}, M \in R^{\left(\gamma+n_{f}\right) \times\left(\gamma-m+n_{f}\right) \text { が存在する }}$ 21).

$$
N B_{a}=0, C_{a} M=0, N M=I_{\gamma-m+n_{f}}
$$

ここで, つぎの正則変換：

$$
\left[\begin{array}{l}
\boldsymbol{y}_{a}(t) \\
\boldsymbol{x}_{2}(t)
\end{array}\right]=T \boldsymbol{z}_{a}(t), T=\left[\begin{array}{c}
C_{a} \\
N
\end{array}\right]
$$

を考えると，拡張系 $(4.2)$ は，

$$
\begin{aligned}
& \dot{\boldsymbol{y}}_{a}(t)=\Theta_{y} \boldsymbol{y}_{a}(t)+B_{c} \widetilde{B}(\boldsymbol{y}) \boldsymbol{u}(t)+C_{2} \boldsymbol{x}_{2}(t) \\
& \dot{\boldsymbol{x}}_{2}(t)=A_{2} \boldsymbol{x}_{2}(t)+B_{2} \boldsymbol{y}_{a}(t)+B_{3} \widetilde{\boldsymbol{a}}\left(\boldsymbol{z}_{\xi}, \boldsymbol{z}_{q}\right) \\
& \dot{\boldsymbol{z}}_{q}(t)=\boldsymbol{q}\left(\boldsymbol{z}_{\xi}, \boldsymbol{z}_{q}\right)
\end{aligned}
$$

と表される。ここに，

$$
\begin{aligned}
& \Theta_{y}=C_{a} A_{a} B_{a} B_{c}^{-1}, B_{c}=C_{a} B_{a}, C_{2}=C_{a} A_{a} M \\
& A_{2}=N A_{a} M, B_{2}=N A_{a} B_{a} B_{c}^{-1}, B_{3}=N B_{1}
\end{aligned}
$$

である。また， $A_{2}$ は，システム $\left(A_{a}, B_{a}, C_{a}\right)$ の零点に対 応する行列であり, $\left(A_{a}, B_{a}, C_{a}\right)$ が最小位相であること 加安定行列となる。すなわち, 任意の正定対称行列 $Q_{2} \in R^{\left(\gamma-m+n_{f}\right) \times\left(\gamma-m+n_{f}\right)}$ に対し，つぎのリアプノフ方 程式:

$$
A_{2}^{T} P_{2}+P_{2} A_{2}=-Q_{2}
$$

を満足する正定対称行列 $P_{2}$ が必ず存在する，このとき，拡張 系の OFEP 性に関して以下の定理が成立する。

《定理 2》仮定 2 を満足するシステム (2.1) に対し, (4.1) で与えられる $\mathrm{PFC}$ を付加して得られる拡張系 (4.2) を考え る。このとき, $\mathrm{PFC}(4.1)$ が拡張系の線形部 $\left(A_{a}, B_{a}, C_{a}\right)$ が ASPRであり, かつ, (4.4) および (4.8)で与えられる $N, M$ および $P_{2}, Q_{2}$ に関し，

$$
\begin{aligned}
\lambda_{\text {min }}\left[Q_{2}\right]> & 2 L_{2}\left\|B_{3}\right\|\left\|P_{2}\right\|\|M\| \\
& +\frac{\left(\alpha_{2} L_{1}\|M\|+2 L_{2}\left\|B_{3}\right\|\left\|P_{2}\right\|\right)^{2}}{4 \delta_{1}}
\end{aligned}
$$

が满足されるように設計されていれば，拡張系 (4.2) は，出 カフィードバック指数受動 (OFEP) となる.ここに, $L_{1}, L_{2}$ は，それぞれ $\boldsymbol{q}\left(\boldsymbol{z}_{\xi}, \boldsymbol{z}_{q}\right)$ および $\widetilde{a}\left(\boldsymbol{z}_{\xi}, \boldsymbol{z}_{q}\right)$ に関する Lipschitz 
定数であり， $\alpha_{2}$ は, ゼロダイナミクスの指数安定性に関連し て (3.12) で与えられる正定数であり，さらに， $\delta_{1}$ は，(3.12) で与えられる正定数 $\alpha_{1}$ に対し, $0<\delta_{1}<\alpha_{1}$ となる正定数で ある。

（証明） PFCの設計条件より，構成された拡張系 (4.2) の線形部 $\left(A_{a}, B_{a}, C_{a}\right)$ は ASPR である. よって, 正則変換 (4.5)により，(4.6)の標準形で表すことができる．このとき， 制御入力を

$$
\boldsymbol{u}(t)=-\widetilde{B}(\boldsymbol{y})^{-1} K \boldsymbol{y}_{a}(t)+-\widetilde{B}(\boldsymbol{y})^{-1} \boldsymbol{v}(t)
$$

と構成すると, 得られる閉ループ系は, (4.6)より

$$
\begin{aligned}
& \dot{\boldsymbol{y}}_{a}(t)=\Theta_{y} \boldsymbol{y}_{a}(t)+B_{c} K \boldsymbol{y}_{a}(t)+B_{c} \boldsymbol{v}(t)+C_{2} \boldsymbol{x}_{2}(t) \\
& \dot{\boldsymbol{x}}_{2}(t)=A_{2} \boldsymbol{x}_{2}(t)+B_{2} \boldsymbol{y}_{a}(t)+B_{3} \tilde{\boldsymbol{a}}\left(\boldsymbol{z}_{\xi}, \boldsymbol{z}_{q}\right) \\
& \dot{\boldsymbol{z}}_{q}(t)=\boldsymbol{q}\left(\boldsymbol{z}_{\xi}, \boldsymbol{z}_{q}\right)
\end{aligned}
$$

と表される.このとき，仮定 2 および $\mathrm{PFC}$ の設計条件より， (3.12) を満足する正定值関数：W( $\left.z_{q}\right)$ および $(4.8)$ を满足す る正定対称行列 $P_{2}, Q_{2}$ が必ず存在することから，つぎの正 定値関数:

$$
V_{a}\left(\boldsymbol{y}_{a}, \boldsymbol{x}_{2}, \boldsymbol{z}_{q}\right)=W\left(\boldsymbol{z}_{q}\right)+\boldsymbol{x}_{2}^{T} P_{2} \boldsymbol{x}_{2}+\frac{1}{2} \boldsymbol{y}_{a}^{T} B_{c}^{-1} \boldsymbol{y}_{a} \text { (4.12) }
$$

を考える.ここで，仮定 $2(1)$ および $(3.12)$ より，W( $\left.\boldsymbol{z}_{q}\right)$ の 時間微分は, $q\left(z_{\xi}, z_{q}\right)$ の Lipschitz 定数を $L_{1}$ とすると

$$
\dot{W}\left(z_{q}\right) \leq-\alpha_{1}\left\|z_{q}\right\|^{2}+\alpha_{2} L_{1}\left\|z_{q}\right\|\left\|z_{\xi}\right\|
$$

と評価できる (付録 $\mathrm{A}$ 参照)。さらに, (4.4), (4.5)より,

$$
T^{-1}=\left[B_{a} B_{c}^{-1}, M\right]
$$

であることから，

$$
\left\|\boldsymbol{z}_{\xi}\right\| \leq\left\|\boldsymbol{z}_{a}\right\| \leq\|M\|\left\|\boldsymbol{x}_{2}\right\|+\left\|B_{a} B_{c}^{-1}\right\|\left\|\boldsymbol{y}_{a}\right\|
$$

と評価できる.よって, $(4.13),(4.15) よ り$,

$$
\dot{W}\left(\boldsymbol{z}_{q}\right) \leq-\alpha_{1}\left\|\boldsymbol{z}_{q}\right\|^{2}+\beta_{1}\left\|\boldsymbol{z}_{q}\right\|\left\|\boldsymbol{x}_{2}\right\|+\beta_{2}\left\|\boldsymbol{z}_{q}\right\|\left\|\boldsymbol{y}_{a}\right\| \text { (4.16) }
$$

を得る.ここに，

$$
\beta_{1}=\alpha_{2} L_{1}\|M\|, \beta_{2}=\alpha_{2} L_{1}\left\|B_{a} B_{c}^{-1}\right\|
$$

である。つぎに, $\boldsymbol{x}_{2}^{T} P_{2} \boldsymbol{x}_{2}$ の時間微分は, (4.8), (4.11)より,

$$
\begin{aligned}
\frac{d}{d t}\left[\boldsymbol{x}_{2}^{T} P_{2} \boldsymbol{x}_{2}\right] \leq & -\lambda_{\min }\left[Q_{2}\right]\left\|\boldsymbol{x}_{2}\right\|^{2}+2\left\|B_{2}\right\|\left\|P_{2}\right\|\left\|\boldsymbol{x}_{2}\right\|\left\|\boldsymbol{y}_{a}\right\| \\
& +2\left\|B_{3}\right\|\left\|P_{2}\right\|\left\|\boldsymbol{x}_{2}\right\|\left\|\widetilde{\boldsymbol{a}}\left(\boldsymbol{z}_{\xi}, \boldsymbol{z}_{q}\right)\right\|
\end{aligned}
$$

と評価できる.ここで, 仮定 $2(2)$ より， $\widetilde{a}\left(z_{\xi}, z_{q}\right)$ に関する Lipschitz 定数を $L_{2}$ とおくと, $(4.15),(4,18)$ より

$$
\begin{aligned}
\frac{d}{d t}\left[\boldsymbol{x}_{2}^{T} P_{2} \boldsymbol{x}_{2}\right] \leq & -\alpha_{Q}\left\|\boldsymbol{x}_{2}\right\|^{2}+\beta_{3}\left\|\boldsymbol{x}_{2}\right\|^{2} \\
& +\beta_{4}\left\|\boldsymbol{x}_{2}\right\|\left\|\boldsymbol{z}_{q}\right\|+2 \beta_{5}\left\|\boldsymbol{x}_{2}\right\|\left\|\boldsymbol{y}_{a}\right\|
\end{aligned}
$$

となる.ここに，

$$
\begin{aligned}
& \alpha_{Q}=\lambda_{\min }[Q] \\
& \beta_{3}=2 L_{2}\left\|B_{3}\right\|\left\|P_{2}\right\|\|M\| \\
& \beta_{4}=2 L_{2}\left\|B_{3}\right\|\left\|P_{2}\right\| \\
& \beta_{5}=2 L_{2}\left\|B_{3}\right\|\left\|P_{2}\right\|\left\|B_{a} B_{c}^{-1}\right\|+2\left\|B_{2}\right\|\left\|P_{2}\right\|
\end{aligned}
$$

である.さらに, $\frac{1}{2} \boldsymbol{y}_{a}^{T} B_{c}^{-1} \boldsymbol{y}_{a}$ の時間微分は, (4.11)より,

$$
\begin{array}{r}
\frac{1}{2} \frac{d}{d t}\left[\boldsymbol{y}_{a}^{T} B_{c}^{-1} \boldsymbol{y}_{a}\right] \leq-\left(\alpha_{K}-\beta_{6}\right)\left\|\boldsymbol{y}_{a}\right\|^{2} \\
\beta_{7}\left\|\boldsymbol{x}_{2}\right\|\left\|\boldsymbol{y}_{a}\right\|+\boldsymbol{y}_{a}^{T} \boldsymbol{v}
\end{array}
$$

と評価できるここに，

$$
\begin{aligned}
& \alpha_{K}=\frac{1}{2} \lambda_{\min }\left[K^{T}+K\right] \\
& \beta_{6}=\left\|\Theta_{y}\right\|\left\|B_{c}^{-1}\right\|, \beta_{7}=\left\|C_{2}\right\|\left\|B_{c}^{-1}\right\|
\end{aligned}
$$

である. 結局, 以上のことより, $V_{a}\left(\boldsymbol{z}_{q}, \boldsymbol{x}_{2}, \boldsymbol{y}_{a}\right)$ の時間微 分は,

$$
\begin{aligned}
\dot{V}_{a} \leq & -\alpha_{1}\left\|\boldsymbol{z}_{q}\right\|^{2}-\left(\alpha_{Q}-\beta_{3}\right)\left\|\boldsymbol{x}_{2}\right\|^{2} \\
& +\left(\beta_{1}+\beta_{4}\right)\left\|\boldsymbol{z}_{q}\right\|\left\|\boldsymbol{x}_{2}\right\|+\beta_{2}\left\|\boldsymbol{z}_{q}\right\|\left\|\boldsymbol{y}_{a}\right\| \\
& +\left(\beta_{5}+\beta_{7}\right)\left\|\boldsymbol{x}_{2}\right\|\left\|\boldsymbol{y}_{a}\right\|-\left(\alpha_{K}-\beta_{6}\right)\left\|\boldsymbol{y}_{a}\right\|^{2} \\
& +\boldsymbol{y}_{a}^{T} \boldsymbol{v} \\
\leq & -\left(\alpha_{1}-\delta_{1}-\delta_{2}\right)\left\|\boldsymbol{z}_{q}\right\|^{2} \\
& -\left(\alpha_{Q}-\beta_{3}-\frac{\left(\beta_{1}+\beta_{4}\right)^{2}}{4 \delta_{1}}-\delta_{3}\right)\left\|\boldsymbol{x}_{2}\right\|^{2} \\
& -\left(\alpha_{K}-\beta_{6}-\frac{\beta_{2}^{2}}{4 \delta_{2}}-\frac{\left(\beta_{5}+\beta_{7}\right)^{2}}{4 \delta_{3}}\right)\left\|\boldsymbol{y}_{a}\right\|^{2} \\
& +\boldsymbol{y}_{a}^{T} \boldsymbol{v}
\end{aligned}
$$

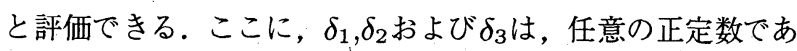
る.このとき, $\delta_{1}$ を $0<\delta_{1}<\alpha_{1}, \delta_{2}$ を $0<\delta_{2}<\alpha_{1}-\delta_{1}$ とと り, さらに, PFCの設計条件 (4.9) より

$$
\alpha_{Q}-\beta_{3}-\frac{\left(\beta_{1}+\beta_{4}\right)^{2}}{4 \delta_{1}}>0
$$

であることから,$\delta_{3} を$

$$
0<\delta_{3}<\alpha_{Q}-\beta_{3}-\frac{\left(\beta_{1}+\beta_{4}\right)^{2}}{4 \delta_{1}}
$$

ととると，

$$
\alpha_{K}>\beta_{6}+\frac{\beta_{2}^{2}}{4 \delta_{2}}+\frac{\left(\beta_{5}+\beta_{7}\right)^{2}}{4 \delta_{3}}
$$

を満たすすべての $K$ に対して $\dot{V}_{a}$ は, ある正定数 $c_{1}, c_{2}, c_{3}$ が存 在して

$$
\dot{V}_{a} \leq-c_{1}\left\|\boldsymbol{z}_{q}\right\|^{2}-c_{2}\left\|\boldsymbol{x}_{2}\right\|^{2}-c_{3}\left\|\boldsymbol{y}_{a}\right\|^{2}+\boldsymbol{y}_{a}^{T} \boldsymbol{v} \text { (4.26) }
$$

と評価できる、これより,

$$
\begin{aligned}
& V_{a}\left(\boldsymbol{y}_{a}(t), \boldsymbol{x}_{2}(t), \boldsymbol{z}_{q}(t)\right)-V_{a}\left(\boldsymbol{y}_{a}(0), \boldsymbol{x}_{2}(0), \boldsymbol{z}_{q}(0)\right) \\
& \leq \int_{0}^{t} \boldsymbol{y}_{a}^{T} \boldsymbol{v} d \tau-\int_{0}^{t}\left(c_{1}\left\|\boldsymbol{z}_{q}\right\|^{2}+c_{2}\left\|\boldsymbol{x}_{2}\right\|^{2}+c_{3}\left\|\boldsymbol{y}_{a}\right\|^{2}\right) d \tau
\end{aligned}
$$
が得られる.よって, (3.12),(4.12) および上式より, 定義 2 の条件が渵足されることから, 出力フィードバック指数受動 であることがわかる。

\subsection{PFC $の$ 具体的設計法}

前節では，PFCの基本的な設計条件を明らかにしたが，問 題はそのような条件を满足するような PFCがいかにして設 計できるかである。ここでは，前節で与えられた設計条件を 满足する具体的な PFCの設計法の提案を行う.

いま, 相対次数 $\left\{\gamma_{1}, \gamma_{2}, \cdots, \gamma_{m}\right\}$ を持つ仮定 2 を満足する システムの標準形 $(2.8)$ を考える. (2.8),(2.11) より このシ 
ステムの $i$ 番目の出力 $y_{i}(t)$ は，それぞれ，つぎのサブシス テムの出力として表すことができる.

$$
\begin{aligned}
\dot{\boldsymbol{z}}_{\xi i}(t) & =A_{i} \boldsymbol{z}_{\xi i}(t)+\boldsymbol{b}_{i} \widetilde{a}_{i}\left(z_{\xi}, \boldsymbol{z}_{q}\right)+\boldsymbol{b}_{i} \widetilde{\boldsymbol{b}}_{i}(\boldsymbol{y})^{T} \boldsymbol{u}(t) \\
\boldsymbol{y}_{i}(t) & =\boldsymbol{c}_{\boldsymbol{i}}^{T} \boldsymbol{z}_{\xi i}(t), \quad i=1, \cdots, m .
\end{aligned}
$$

ここに，

$$
\begin{aligned}
& {\left[\boldsymbol{z}_{\xi 1}(t)^{T}, \boldsymbol{z}_{\xi 2}(t)^{T}, \cdots, \boldsymbol{z}_{\xi m}(t)^{T}\right]^{T}=\boldsymbol{z}_{\xi}(t)} \\
& {\left[\widetilde{a}_{1}\left(\boldsymbol{z}_{\xi}, \boldsymbol{z}_{q}\right), \widetilde{a}_{2}\left(\boldsymbol{z}_{\xi}, \boldsymbol{z}_{q}\right), \cdots, \widetilde{a}_{m}\left(\boldsymbol{z}_{\xi}, \boldsymbol{z}_{q}\right)\right]^{T}=\widetilde{\boldsymbol{a}}\left(\boldsymbol{z}_{\xi}, \boldsymbol{z}_{q}\right)} \\
& {\left[\widetilde{\boldsymbol{b}}_{1}(\boldsymbol{y}), \widetilde{\boldsymbol{b}}_{2}(\boldsymbol{y}), \cdots, \widetilde{\boldsymbol{b}}_{m}(\boldsymbol{y})\right]^{T}=\widetilde{B}(\boldsymbol{y})}
\end{aligned}
$$

である. そこで, 各サブシステムに対し，それぞれつぎの $n_{f i}$ 次の PFCを付加する.

$$
\begin{aligned}
& \dot{\boldsymbol{z}}_{f i}(t)=A_{f i} \boldsymbol{z}_{f i}(t)+\boldsymbol{b}_{f i} \widetilde{\boldsymbol{b}}_{i}(\boldsymbol{y})^{T} \boldsymbol{u}(t) \\
& \boldsymbol{y}_{f i}(t)=c_{f i}^{T} \boldsymbol{z}_{f i}(t)
\end{aligned}
$$

ここに, $\left(A_{f i}, \boldsymbol{b}_{f i}, \boldsymbol{c}_{f i}\right)$ は，伝達関数表現で

$$
F_{i}(s)=c_{f i}^{T}\left(s I-A_{f i}\right) b_{f i}=\frac{\delta_{i}^{\gamma_{i}} b_{i}\left(\delta_{i} s\right)}{\alpha_{f i} a_{i}\left(\delta_{i} s\right)}
$$

と表すことができるように与えるものとする．ただし， $\delta_{i}$ は， 任意の正定数であり, $a_{i}(s), b_{i}(s)$ および $\alpha_{f i}$ は, 以下の設計 条件を满足しているものとする。

$\left[a_{i}(s), b_{i}(s), \alpha_{f i}\right.$ の設計条件]

(1) $\quad a_{i}(s):\left(\gamma_{i}-1\right)$ 次モニック安定多項式

$$
b_{i}(s):\left(\gamma_{i}-2\right) \text { 次モニック多項式 }
$$

(2) $\quad n_{i}(s)=s^{\gamma_{i}} b_{i}(s)+\alpha_{f i} a_{i}(s)$ が安定多項式

(4.29) で与えられる PFCは，具体的には，

$$
\begin{gathered}
a_{i}(s)=s^{\gamma_{i}-1}+a_{i, \gamma_{i}-2} s^{\gamma_{i}-2}+\cdots+a_{i, 1} s+a_{i, 0} \\
b_{i}(s)=s^{\gamma_{i}-2}+b_{i, \gamma_{i}-3} s^{\gamma_{i}-3}+\cdots+b_{i, 1} s+b_{i, 0}
\end{gathered}
$$

とおくと

$$
\begin{aligned}
A_{f i} & =\left[\begin{array}{ccccc}
0 & 1 & 0 & \cdots & 0 \\
0 & 0 & 1 & \cdots & 0 \\
\cdot & \cdot & \cdot & \cdots & \cdot \\
0 & 0 & 0 & \cdots & 1 \\
-\frac{a_{i, 0}}{\delta_{i}^{\gamma_{i}-1}} & -\frac{a_{i, 1}}{\delta_{i}^{\gamma_{i}-2}} & -\frac{a_{i, 2}}{\delta_{i}^{\gamma_{i}-3}} & \cdots & -\frac{a_{i, \gamma_{i}-2}}{\delta_{i}}
\end{array}\right] \\
\boldsymbol{b}_{f i}^{T} & =\left[\begin{array}{lll}
0 & \cdots & 0
\end{array}\right] \\
\boldsymbol{c}_{i}^{T} & =\left[\frac{\delta_{i}}{\alpha_{f i}} b_{i, 0}, \frac{\delta_{i}^{2}}{\alpha_{f i}} b_{i, 1}, \cdots, \frac{\delta_{i}^{\gamma_{i}-2}}{\alpha_{f i}} b_{i, \gamma_{i}-3}, \frac{\delta_{i}^{\gamma_{i}-1}}{\alpha_{f i}}\right]
\end{aligned}
$$

と与えられる。 また, PFC を付加した搪張系は,

$$
\begin{aligned}
& \dot{\boldsymbol{z}}_{a}(t)=A_{a} \boldsymbol{z}_{a}(t)+B_{a} \widetilde{B}(\boldsymbol{y}) \boldsymbol{u}(t)+B_{1} \widetilde{\boldsymbol{a}}\left(\boldsymbol{z}_{\xi}, \boldsymbol{z}_{q}\right) \\
& \dot{\boldsymbol{z}}_{q}(t)=\boldsymbol{q}\left(\boldsymbol{z}_{\xi}, \boldsymbol{z}_{q}\right) \\
& \boldsymbol{y}_{a}(t)=\boldsymbol{y}(t)+\boldsymbol{y}_{f}(t)=C_{a} \boldsymbol{z}_{a}(t)
\end{aligned}
$$

と表される.ここに，

$$
\begin{aligned}
& \boldsymbol{z}_{a}(t)=\left[\boldsymbol{z}_{a 1}(t)^{T}, \cdots, \boldsymbol{z}_{a m}(t)^{T}\right]^{T} \\
& \boldsymbol{z}_{a i}(t)=\left[\boldsymbol{z}_{\xi i}(t)^{T}, \boldsymbol{z}_{f i}(t)^{T}\right]^{T}
\end{aligned}
$$

であり，

$$
\begin{aligned}
& A_{a}=\operatorname{diag}\left[A_{a i}\right], A_{a i}=\left[\begin{array}{cc}
A_{i} & 0 \\
0 & A_{f i}
\end{array}\right] \\
& B_{a}=\operatorname{diag}\left[\boldsymbol{b}_{a i}\right], \boldsymbol{b}_{a i}=\left[\begin{array}{c}
\boldsymbol{b}_{i} \\
\boldsymbol{b}_{f i}
\end{array}\right] \\
& C_{a}=\operatorname{diag}\left[\boldsymbol{c}_{a i}^{T}\right], \boldsymbol{c}_{a i}^{T}=\left[\boldsymbol{c}_{i}^{T}, \boldsymbol{c}_{f i}^{T}\right] \\
& B_{1}=\operatorname{diag}\left[\boldsymbol{b}_{1 i}\right], \boldsymbol{b}_{1 i}=\left[\begin{array}{c}
\boldsymbol{b}_{i} \\
0
\end{array}\right], i=1, \cdots, m
\end{aligned}
$$

である。このとき，構成された拡張系および PFC 設計に関 して以下の補題が成立する。

[補題 1] 仮定 2 を満足する相対次数 $\left\{\gamma_{1}, \cdots, \gamma_{m}\right\}$ を持つシ ステム (2.1) に対し, 上述の設計条件を満足する $\mathrm{PFC}(4.28)$ を付加した拡張系の線形部 $\left(A_{a}, B_{a}, C_{a}\right)$ はASPR であり,

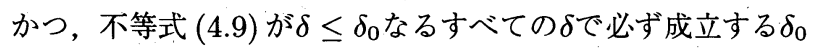
が存在する。

（証明）初めに, 拡張系の線形部 $\left(A_{a}, B_{a}, C_{a}\right)$ が ASPR となることを示す. (2.12),(4.29) および (4.34) より拡張系線 形部 $\left(A_{a}, B_{a}, C_{a}\right)$ に対する伝達関数は

$$
\begin{aligned}
G_{a}(s) & =C_{a}\left(s I-A_{a}\right) B_{a}=\operatorname{diag}\left[G_{i}(s)+F_{i}(s)\right](4.35) \\
G_{i}(s) & =\frac{1}{s^{\gamma_{i}}}, i=1, \cdots, m
\end{aligned}
$$

と表すことができる.よって,$G_{i}(s)+F_{i}(s)$ が ASPR とな ることを示せば十分である.

(4.29),(4.36)より,

$$
G_{i}(s)+F_{i}(s)=\frac{\delta_{i}^{\gamma_{i}} s^{\gamma_{i}} b_{i}\left(\delta_{i} s\right)+\alpha_{f i} a_{i}\left(\delta_{i} s\right)}{\alpha_{f i} s^{\gamma_{i}} a_{i}\left(\delta_{i} s\right)}
$$

を得る. $a_{i}(s), b_{i}(s), \alpha_{f i}$ の設計条件 $(2)$ より，

$$
n_{i}\left(\delta_{i} s\right)=\delta_{i}^{\gamma_{i}} s^{\gamma_{i}} b_{i}\left(\delta_{i} s\right)+\alpha_{f i} a_{i}\left(\delta_{i} s\right)
$$

は, 安定多項式である. よって, $G_{i}(s)+F_{i}(s)$ は, 最小位相 である。さらに， $a_{i}(s), b_{i}(s), \alpha_{f i}$ の設計条件 $(1)$ より,

$$
\begin{aligned}
& \operatorname{deg}\left[s^{\gamma_{i}} a_{i}\left(\delta_{i} s\right)\right]=2 \gamma_{i}-1 \\
& \operatorname{deg}\left[\delta_{i}^{\gamma_{i}} s^{\gamma_{i}} b_{i}\left(\delta_{i} s\right)+\alpha_{f i} a_{i}\left(\delta_{i} s\right)\right]=2 \gamma_{i}-2
\end{aligned}
$$

であることから, $G_{i}(s)+F_{i}(s)$ の相対次数は 1 である.また, 明らかに最高位係数は正であるので, $G_{i}(s)+F_{i}(s)$ は ASPR である.よって, 拡張系線形部 $\left(A_{a}, B_{a}, C_{a}\right)$ は, ASPR と いえる。

つぎに不等式 (4.9) が成立することを示す. 準備として, 拡 張系 $(4.32)-(4.34)$ に対し，つぎの正則変換を考える.

$$
\begin{aligned}
\bar{z}_{a}(t) & =\left[\overline{\boldsymbol{z}}_{a 1}(t)^{T}, \cdots, \overline{\boldsymbol{z}}_{a m}(t)^{T}\right]^{T}=T_{a} z_{a}(t) \\
T_{a} & =\operatorname{diag}\left[T_{a i}\right]_{i=1, \cdots, m}, T_{a i}=\left[\begin{array}{cc}
T_{p i} & 0 \\
0 & T_{f i}
\end{array}\right] \\
T_{p i} & =\operatorname{diag}\left[\frac{1}{\delta_{i}^{\gamma_{i}-1}}, \frac{1}{\delta_{i}^{\gamma_{i}-2}}, \cdots, \frac{1}{\delta_{i}}, 1\right] \\
T_{f i} & =\operatorname{diag}\left[\frac{1}{\delta_{i}^{\gamma_{i}-2}}, \frac{1}{\delta_{i}^{\gamma_{i}-3}}, \cdots, \frac{1}{\delta_{i}}, 1\right]
\end{aligned}
$$

この正則変換により，拡張系 (4.32) は,

$$
\begin{aligned}
& \dot{\overline{\boldsymbol{z}}}_{a}(t)=\bar{A}_{a} \boldsymbol{z}_{a}(t)+\bar{B}_{a} \widetilde{B}(\boldsymbol{y}) \boldsymbol{u}(t)+\bar{B}_{1} \widetilde{\boldsymbol{a}}\left(T_{p}^{-1} \overline{\boldsymbol{z}}_{\xi}, \boldsymbol{z}_{q}\right) \\
& \dot{\boldsymbol{z}}_{q}(t)=\boldsymbol{q}\left(T_{p}^{-1} \overline{\boldsymbol{z}}_{\xi}, \boldsymbol{z}_{q}\right) \\
& \boldsymbol{y}_{a}(t)=\bar{C}_{a} \overline{\boldsymbol{z}}_{a}(t)
\end{aligned}
$$


と表される.ここに，

$$
\begin{aligned}
& \bar{A}_{a}=\operatorname{diag}\left[\bar{A}_{a i}\right], \bar{A}_{a i}=\left[\begin{array}{cc}
\bar{A}_{i} & 0 \\
0 & \bar{A}_{f i}
\end{array}\right] \\
& \bar{A}_{i}=T_{p i} A_{i} T_{p i}^{-1}=\left[\begin{array}{ccc}
0 & \frac{1}{\delta_{i}} I_{\gamma_{i}-1} \\
\vdots & & \\
0 & \cdots & 0
\end{array}\right] \\
& \bar{A}_{f i}=T_{f i} A_{f i} T_{f i}^{-1} \\
& =\left[\begin{array}{ccccc}
0 & \frac{1}{\delta_{i}} & 0 & \cdots & 0 \\
0 & 0 & \frac{1}{\delta_{i}} & \cdots & 0 \\
\cdot & \cdot & \cdot & \cdots & . \\
0 & 0 & 0 & \cdots & \frac{1}{\delta_{i}} \\
-\frac{a_{i, 0}}{\delta_{i}} & -\frac{a_{i, 1}}{\delta_{i}} & -\frac{a_{i, 2}}{\delta_{i}} & \cdots & -\frac{a_{i, \gamma_{i}-2}}{\delta_{i}}
\end{array}\right] \\
& \bar{B}_{a}=\operatorname{diag}\left[\overline{\boldsymbol{b}}_{a i}\right], \overline{\boldsymbol{b}}_{a i}=T_{a i}\left[\begin{array}{c}
\boldsymbol{b}_{i} \\
\boldsymbol{b}_{f i}
\end{array}\right]=\left[\begin{array}{c}
\boldsymbol{b}_{i} \\
\boldsymbol{b}_{f i}
\end{array}\right]
\end{aligned}
$$

$\bar{C}_{a}=\operatorname{diag}\left[\overline{\boldsymbol{c}}_{a i}^{T}\right], \overline{\boldsymbol{c}}_{a i}^{T}=\left[\boldsymbol{c}_{i}^{T}, \boldsymbol{c}_{f i}^{T}\right] T_{a i}^{-1}=\delta_{i}^{\gamma_{i}-1}\left[\boldsymbol{c}_{i}^{T}, \widetilde{\boldsymbol{c}}_{f i}^{T}\right]$

$\widetilde{c}_{f i}^{T}=\frac{1}{\alpha_{f i}}\left[b_{i, 0}, b_{i, 1}, \cdots, b_{i, \gamma_{i}-3}, 1\right]$

$\bar{B}_{1}=\operatorname{diag}\left[\overline{\boldsymbol{b}}_{1 i}\right], \overline{\boldsymbol{b}}_{1 i}=T_{a i} \boldsymbol{b}_{1 i}=\boldsymbol{b}_{1 i}, i=1, \cdots, m$

である。

初めに示したように，拡張系の線形部は ASPR であること から，

$$
\overline{N B}_{a}=0, \bar{C}_{a} \bar{M}=0, \overline{N M}=I_{\gamma-m+n_{f}}
$$

を満足する行列 $\bar{N} \in R^{\left(\gamma-m+n_{f}\right) \times\left(\gamma+n_{f}\right)}, \bar{M} \in$ $R^{\left(\gamma+n_{f}\right) \times\left(\gamma-m+n_{f}\right)}\left(n_{f}=\gamma-m\right)$ が存在し，たとえばつ ぎのように与えることができる21).

$$
\begin{aligned}
& \bar{N}=\operatorname{diag}\left[\bar{N}_{i}\right] \\
& \bar{N}_{i}=\left[\begin{array}{c|c|c}
0 & & \\
\vdots & I_{\gamma_{i}-1} & 0 \\
0 & & \\
-1 / d_{f i} & & -\widetilde{\boldsymbol{c}}_{f i}^{T} / d_{f i} \\
\hline-\boldsymbol{b}_{f i} / d_{f i} & 0 & I_{n_{f i}}-\boldsymbol{b}_{f i} \widetilde{\boldsymbol{c}}_{f i}^{T} / d_{f i}
\end{array}\right]
\end{aligned}
$$

$$
\bar{M}=\operatorname{diag}\left[\bar{M}_{i}\right]
$$

$$
\begin{aligned}
\bar{M}_{i}=\left[\begin{array}{cc}
0 \cdots 0 & -\tilde{\boldsymbol{c}}_{f i}^{T} \\
I_{\gamma_{i}-1} & 0 \\
0 & I_{n_{f i}}
\end{array}\right] \\
\text { ここに, } d_{f i}=\widetilde{\boldsymbol{c}}_{f i}^{T} \boldsymbol{b}_{f i}=1 / \alpha_{f i}, n_{f i}=\gamma_{i}-1 \text { である.この }
\end{aligned}
$$

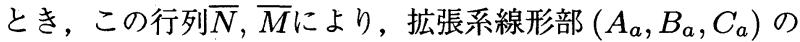
零点に対応する行列 $\bar{A}_{2}$ は,

$$
\bar{A}_{2}=\operatorname{diag}\left[\bar{A}_{2 i}\right]=\operatorname{diag}\left[\delta_{i}^{-1} M_{0 i}\right]
$$

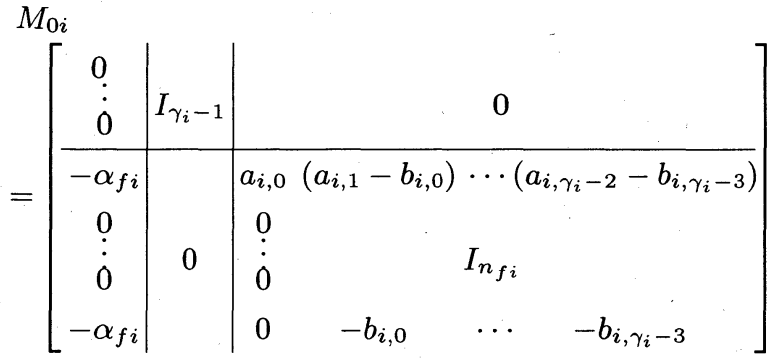

と得られる。 $M_{0 i}$ は， $\delta_{i}$ に無関係な行列となっている．また， 安定多項式 $n_{i}(s)=0$ の根を $-\lambda_{i j}, j=1, \cdots, 2 \gamma_{i}-2$ とおく と, 拡張系線形部の零点多項式が $n_{i}\left(\delta_{i} s\right)$ で与えられること から, $\bar{A}_{2}$ の固有值は，

$$
-\overline{\lambda_{i j}}=-\frac{\lambda_{i j}}{\delta_{i}}, i=1, \cdots, m, j=1, \cdots, 2 \gamma_{i}-2 \text { (4.46) }
$$

で与えられる。このとき，(4.45),(4.56)ょり，

$$
\left\|e^{\bar{A}_{2 i} t}\right\| \leq \bar{m}_{0 i} e^{-\frac{\lambda_{M i}}{\delta_{i}} t}
$$

と評価できる正定数 $\bar{m}_{0 i}$ が存在することが簡単に解る。ここ に, $\lambda_{M i}=\min _{j}\left(\lambda_{i j}\right)$ である.

このとき，この $\bar{A}_{2}$ およびある正定対称行列 $Q_{2}$ に対するリア プノフ方程式 (4.8) を満足する正定対称行列 $P_{2}$ は,

$$
P_{2}=\int_{0}^{\infty} e^{\bar{A}_{2}^{T} t} Q_{2} e^{\bar{A}_{2} t} d t
$$

で与えられ，

$$
Q_{2}=\operatorname{diag}\left[\frac{1}{\delta_{i}} I_{\gamma_{i}-1+n_{f i}}\right]_{i=1, \cdots, m}
$$

とおくと

$$
P_{2}=\operatorname{diag}\left[\frac{1}{\delta_{i}} \int_{0}^{\infty} e^{\bar{A}_{2 i}^{T} t} e^{\bar{A}_{2 i} t} d t\right]_{i=1, \cdots, m}
$$

と表すことができる.よって,このときの $P_{2}$ は,

$$
\left\|P_{2}\right\| \leq \max _{i}\left(\frac{\bar{m}_{0 i}^{2}}{2 \lambda_{M i}}\right)
$$

と評価できる。ささらに,

$$
\left\|\bar{B}_{3}\right\|=\left\|\overline{N B}_{1}\right\|=1,\|\bar{M}\|<\infty
$$

であり, $\boldsymbol{q}\left(T_{p}^{-1} \overline{z_{\xi}}, \boldsymbol{z}_{q}\right), \widetilde{\boldsymbol{a}}\left(T_{p}^{-1} \overline{z_{\xi}}, \boldsymbol{z}_{q}\right)$ の Lipschitz 定数 $\bar{L}_{1}$, $\bar{L}_{2}$ がそれぞれ

$$
\bar{L}_{1}=L_{1}\left\|T_{p}^{-1}\right\|, \quad \bar{L}_{2}=L_{2}\left\|T_{p}^{-1}\right\|
$$

で与えられることを考虑すると，十分小さな $\delta_{i}$ に対して，不 等式 (4.9) が必ず成立することがわかる，すなわち，不等式 (4.9) がある $\delta_{0}$ 以下のすべての $\delta_{i}$ で成立する $\delta_{0}$ が存在すること がわかる。

\section{5. 数值シミュレーション}

ここでは, 簡単な数値シミュレーションにより提案手法の 有効性の検証を行う.

Case-1:多入出力系の特別な場合として，まず，1 入出力系 に関するシミュレーション例を示す．制御対象は，以下のよ うに Output-feedback formで表される 3 次系とする.

$$
\begin{aligned}
\dot{x}_{1} & =\frac{x_{1}^{3}}{1+a_{1} x_{1}^{2}}+x_{2} \\
\dot{x}_{2} & =x_{3}+\sin \left(c_{1} x_{1}\right) x_{1}+b_{1}\left(1+x_{1}^{2}\right) u \\
\dot{x}_{3} & =\cos \left(c_{2} x_{1}\right) \frac{x_{1}}{1+a_{2} x_{1}^{2}}+b_{2}\left(1+x_{1}^{2}\right) u \\
y & =x_{1}
\end{aligned}
$$

ここで, $a_{1}=3, a_{2}=4, b_{1}=1, b_{2}=2$ および $c_{1}=c_{2}=2$ は未知とする.また実際の次数 (3 次) も未知とする. ただし, 


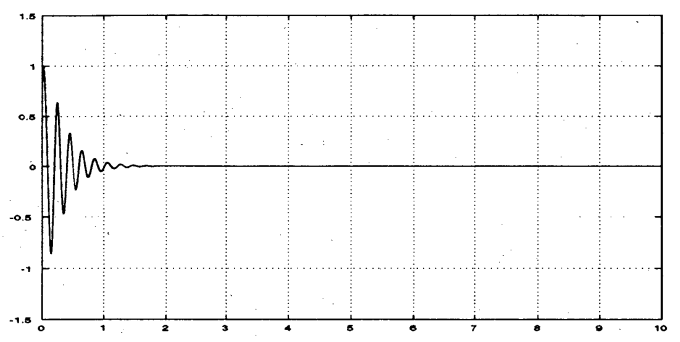

Fig. 1 Simulation results (Case-1): plant output

最小位相であり，相対次数が 2 であることは事前情報として わかっているものとする. 上記システムは, 非線形項が未知パ ラメー夕に関して線形でないため, 従来の適応 Backstepping 法 ${ }^{22)}$ は適応できない.

さて，このシステムは既知であれば， $z_{1}=x_{1}, z_{2}=$ $\frac{z_{1}^{3}}{1+3 z_{1}^{2}}+x_{2}, z_{3}=x_{3}-2 z_{2}$ なる正則変換により,

$$
\begin{aligned}
\dot{z}_{1}= & z_{2} \\
\dot{z}_{2}= & 2 z_{2}+z_{3}+\frac{3\left(1+z_{1}^{2}\right) z_{1}^{2}}{\left(1+3 z_{1}^{2}\right)^{2}} z_{2} \\
& +\sin \left(2 z_{1}\right) z_{1}+\left(1+z_{1}^{2}\right) u \\
\dot{z}_{3}= & -2 z_{3}-4 z_{2}+\cos \left(2 z_{1}\right) \frac{z_{1}}{1+4 z_{1}^{2}} \\
& -6 \frac{\left(1+z_{1}^{2}\right) z_{1}^{2}}{\left(1+3 z_{1}^{2}\right)^{2}} z_{2}-2 \sin \left(2 z_{1}\right) z_{1}
\end{aligned}
$$

と変形できる.この変形において, $\widetilde{B}(y)=1+y^{2}$ の情報は, 元のシステムが不確かであっても $x_{2}$-システムの入力項の非 線形項に関する情報のみで得られることがわかる，また，実 際には，最小位相で非線形項が $y$ に関して Lipshitzであるこ とがわかっていれば， $x_{3}$ 以下のシステムに関するその他の情 報は提案手法の制御系設計には必要がないこともわかる（た とえ次数が 4 次や 5 次であっても同様の变形で $z_{2}$-システ ムには関係なく 3 次以降のシステム（ゼロダイナミクス）が 得られるため)．このシステムは相対次数 2 を持つことから, つぎの PFC :

$$
\dot{z}_{f}=-\frac{a_{f 1}}{\delta_{1}} z_{f}+\left(1+y^{2}\right) u, \quad y_{f}=\frac{\delta_{1}}{\alpha_{f 1}} z_{f}
$$

を導入する.ここでは， $a_{f 1}=1, \alpha_{f 1}=10, \delta_{1}=0.1$ と与 えた. 初期值 $\boldsymbol{x}(0)=[1,0.5,0.1]^{T}$ に対し, シミュレーション を行った結果を Fig. 1 Fig. 3 に示す. なお，適応調整則 (3.5) の設計パラメータは， $\Gamma=1000$ と与えている．また， Fig. 4 は，PFC を付加しなかった場合の結果である（この 場合は，制御系の安定性は保証されていない.)。これらの結 果より，PFCの導入によって良好な結果が得られていること がわかる。

Case:2つぎのように与えられる 2 入出力非線形系を考える.

$$
\dot{\boldsymbol{x}}=\left[\begin{array}{c}
-x_{1}+3 x_{5}^{6}+2 x_{3}^{2} \\
\sin x_{3} \\
x_{2}+x_{3} \\
\sin x_{5} \\
x_{4}+x_{5}
\end{array}\right]
$$

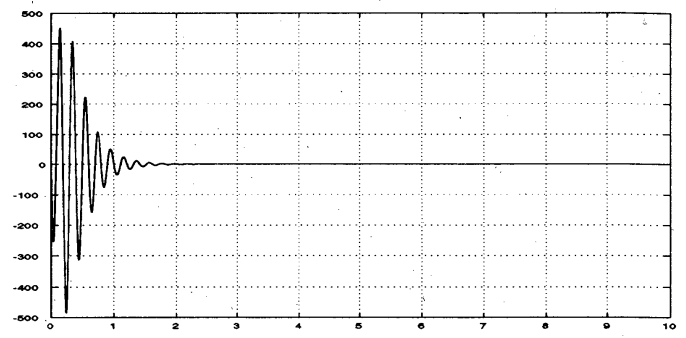

Fig. 2 Simulation results (Case-1): control input

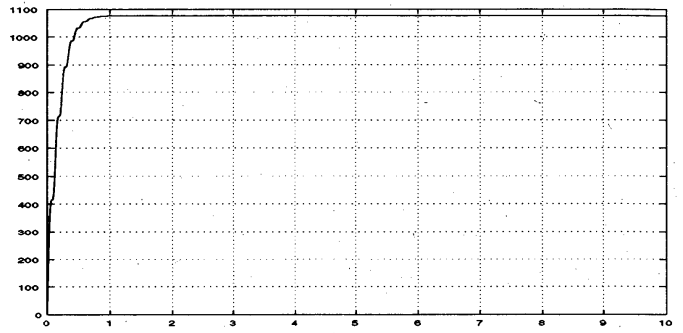

Fig. 3 Simulation results (Case-1): estimated value of $K(t)$

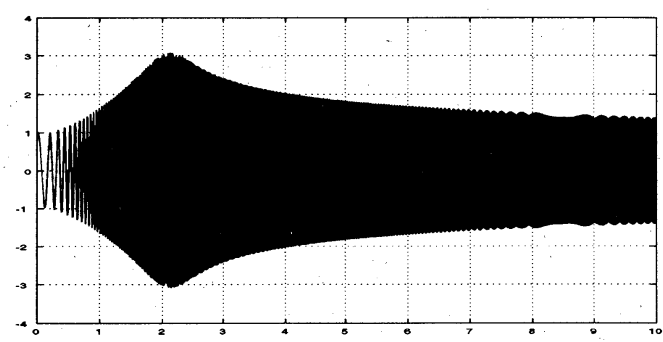

Fig. 4 Simulation results without PFC (Case-1): plant output

$$
\begin{aligned}
& +\left[\begin{array}{cc}
0 & 0 \\
\exp \left(x_{3}+x_{5}\right)+2 & \exp x_{3} \\
0 & 0 \\
\exp x_{5} & \left(\exp x_{3}+\exp x_{5}\right)^{2} \\
0 & 0
\end{array}\right] \boldsymbol{u} \\
& \boldsymbol{y}=\left[\begin{array}{ll}
x_{3} & x_{5}
\end{array}\right]^{T}
\end{aligned}
$$

このとき，つぎの正則変換：

$$
\begin{aligned}
& z_{1}=x_{3} \\
& z_{2}=x_{2}+x_{3} \\
& z_{3}=x_{5} \\
& z_{4}=x_{4}+x_{5} \\
& z_{5}=x_{1}
\end{aligned}
$$

を考えると，(5.4)の標準形は，

$$
\begin{aligned}
& \dot{\boldsymbol{z}}_{\xi}=A \boldsymbol{z}_{\xi}+B \widetilde{\widetilde{a}}\left(\boldsymbol{z}_{\xi}, z_{q}\right)+B \widetilde{B}(\boldsymbol{y}) \boldsymbol{u} \\
& \dot{z}_{q}=-z_{q}+3 z_{3}^{6}+2 z_{1}^{2} \\
& \boldsymbol{y}=\boldsymbol{c}^{T} \boldsymbol{z}_{\xi}
\end{aligned}
$$

と表される。ここに， 


$$
\begin{aligned}
& \boldsymbol{z}_{\xi}=\left[z_{1}, z_{2}, z_{3}, z_{4}\right]^{T}, z_{q}=z_{5} \\
& A=\left[\begin{array}{llll}
0 & 1 & 0 & 0 \\
0 & 0 & 0 & 0 \\
0 & 0 & 0 & 1 \\
0 & 0 & 0 & 0
\end{array}\right], B=\left[\begin{array}{ll}
0 & 0 \\
1 & 0 \\
0 & 0 \\
0 & 1
\end{array}\right], \boldsymbol{c}^{T}=\left[\begin{array}{lll}
1 & 0 & 1
\end{array}\right] \\
& \widetilde{\boldsymbol{a}}\left(\boldsymbol{z}_{\xi}, z_{q}\right)=\left[\begin{array}{cc}
\sin z_{1}+z_{2} \\
\sin z_{3}+z_{4}
\end{array}\right] \\
& \widetilde{B}(\boldsymbol{y})=\left[\begin{array}{cc}
\exp \left(z_{1}+z_{3}\right)+2 & \exp z_{1} \\
\exp z_{3} & \left(\exp z_{1}+\exp z_{3}\right)^{2}
\end{array}\right]
\end{aligned}
$$

である。

Fig. 5 は，初期值を $\boldsymbol{x}(0)=[2.5,0.5,2,-2,1]$ と与えたと きの自由応答の結果である. 対象システムは不安定系である ので発散していることがわかる。

さて, 対象システムは, 指数最小位相であるが相対次数 $\{2,2\}$ を持うことからつぎの PFC 導入しする.

$$
\begin{aligned}
& \dot{\boldsymbol{z}}_{f}=\left[\begin{array}{cc}
-\frac{a_{f 1}}{\delta_{1}} & 0 \\
0 & -\frac{a_{f 2}}{\delta_{2}}
\end{array}\right] \boldsymbol{z}_{f}+\widetilde{B}(\boldsymbol{y}) \boldsymbol{u} \\
& \boldsymbol{y}_{f}=\left[\begin{array}{cc}
\frac{\delta_{1}}{\alpha_{f 1}} & 0 \\
0 & \frac{\delta_{2}}{\alpha_{f 2}}
\end{array}\right] \boldsymbol{z}_{f}
\end{aligned}
$$

ここでは,

$$
a_{f 1}=a_{f 2}=3, \alpha_{f 1}=\alpha_{f 2}=2 / 3, \delta_{1}=\delta_{2}=0.06
$$

と与え拡張系の OFEP 化を図った。

初期值 $\boldsymbol{x}(0)=[2.5,0.5,2,-2,1]$ に対し，適応調整則 $(3.5)$ において, $\Gamma=\operatorname{diag}\left[5 \times 10^{3}, 5 \times 10^{4}\right]$ と与えたときの制御結 果を Fig. 6〜Fig. 8 に示す. PFC 導入によるバイアス効果 の影響もなく良好な結果が得られている。

\section{6. 結 言}

本報告では, 多入出力 affine 非線形システムに対して, 出 カフィードバック指数受動性 (OFEP) のもと出力フィード バック形式のシステムの次数に依存しない適応制御系が簡単 に構成できることを示した。さらに，対象システムが OFEP 条件を满足していない場合に対して，この OFEP 条件を緩 和するための非線形系に対する $\mathrm{PFC}$ 設計条件および導出さ れた設計条件を満足するための具体的な PFC 設計法法の提 案を行った。また，簡単な数值シミュレーションにより提案 手法の有効性の検証を行った.

\section{参 考 文 献}

1) C. I. Byrnes, A. Isidori and J. Willems: Passivity, Feedback Equivalence, and the Global Stabilization of Minimum Phase Nonlinear Systems, IEEE Trans, Automatic Control, 36-11, 1228/1240(1991)

2) D. J. Hill and P. J. Moylan: Stability Results for Nonlinear Feedback Systems, Automatica, bf 13, 377/382(1977)

3) M. Krstic, I. Kanellakopoulos and P. V. Kokotovic: Passivity and Parametric Robustness of a New Class of Adaptive Systems, Automatica, 30-11, 1703/1716(1994)

4) H. K. Khalil:Nonlinear Systems, 2nd ed., Prentice Hall (1996)

5) M. M. Seron, D. J. Hill and A. L. Fradkov: Nonlinear

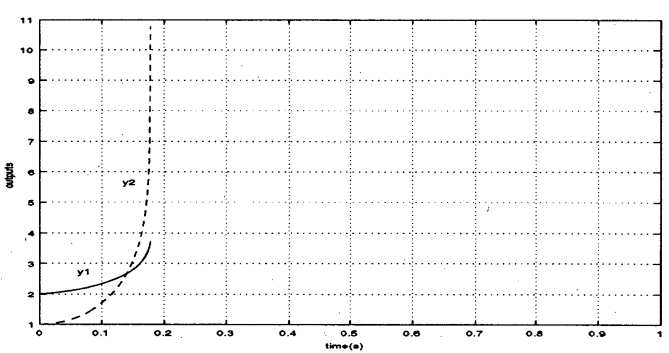

Fig. 5 Free response of the controlled plant

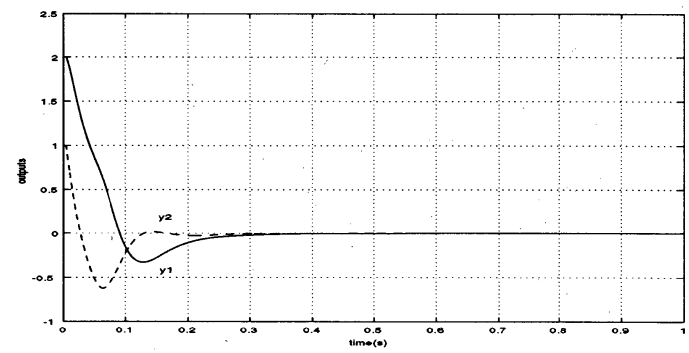

Fig. 6 Simulation results (Case-2): plant outputs

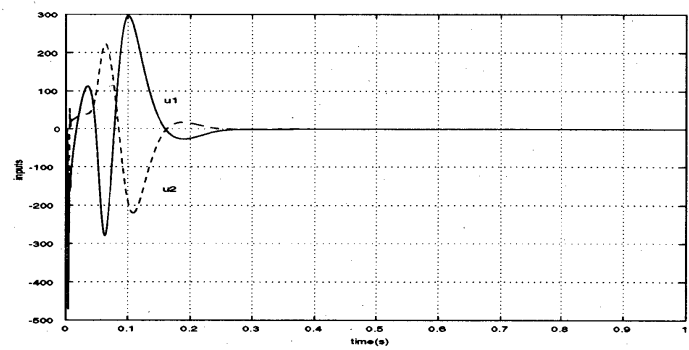

Fig. 7 Simulation results (Case-2): control inputs

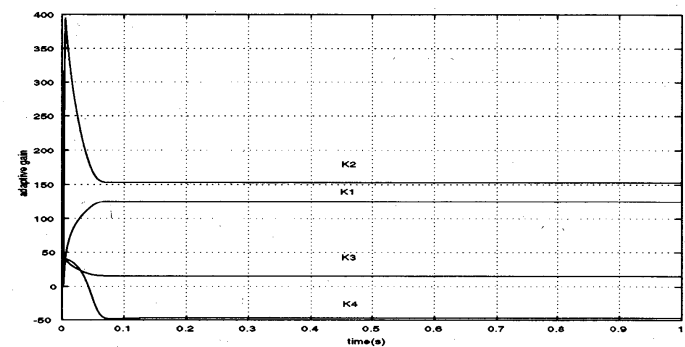

Fig. 8 Simulation results (Case-2): estimated values of $K(t)$

Adaptive Control of Feedback Passive System, Automatica, 31-7, 1053/1060(1995)

6) P. V. Kokotovic, M. Kristic and I. Kanellakopoulos: Backstepping to Passivity: Recursive Design of Adaptive Systems, Proc. of 31st IEEE CDC, 3276/3280(1992)

7) I. Bar-Kana: Positive Realness in Multivariable Stationary Linear Systems, Journal of the Franklin Institute, $\mathbf{3 2 8}$, 403/417(1991)

8) H. Kaufman, I. Bar-Kana and K. Sobel: Direct Adaptive Control Algorithms, 2nd ed., Springer-Verlag (1998)

9) I. Bar-Kana: Parallel Feedforward and Simplified Adaptive Control, Journal of Adaptive Control and Signal Processing, 1, 95/109(1987)

10) Z. Iwai and I. Mizumoto: Realization of Simple Adaptive 
Control by Using Parallel Feedforward Compensator, Int. J. Control, 59-6, 1543/1565(1994)

11) I. Mizumoto and Z. Iwai: Simplified Adaptive Model Output Following Control for Plants with Unmodelled Dynamics, Int. J. Control, 64-1, 61/80(1996)

12) A. L. Fradkov: Adaptive stabilization for minimum-phase multi-input plants without output derivatives measurement, Physics-Doklady, 39-8, 550/552(1994)

13) A. L. Fradkov: Shunt Output Feedback Adaptive Controllers for Nonlinear Plants, 13th IFAC World Congress, San Francisco, K, 367/372(1996)

14) M.V. Druzhinina, A.L. Fradkov: Reduced order shunt nonlinear adaptive controller, Proc. 4th European Control Conference (1997)

15) A. L. Fradkov, I. Mizumoto and Z. Iwai: Shunt Output Feedback Adaptive Tracking for Nonlinear Plants, Proc. of the 6th St. Petersburg Symp. on Adaptive Systems Theory (SPAS'99), 79/85 (1999)

16) A. L. Fradkov and D. J. Hill: Exponential Feedback Passivity and Stabilizability of Nonlinear Systems, Automatica, 34-6, 697/703(1998)

17) C. I. Byrnes and A. Isidori: Asymptotic Stabilization on Minimum Phase Nonlinear Systems, IEEE Trans, Automatic Control, 36-10, 1122/1137(1991)

18) A. Isidori: Nonlinear Control Systems, 3rd ed., SpringerVerlag (1998)

19) N. N. Krasovskii: Stability of Motion, Stanford (1963)

20) S. Sastry and M. Bodson: Adaptive Control, Prentice Hall (1989)

21) B. Kouvaritakis and MacFarlane: Geometric Approach to Analysis and Synthesis of System Zeros; Part 1. Square Systems, Int. J. Control, 23-2, 146/166 (1976)

22) M. Krstic, I. Kanellakopoulos and P.V. Kokotovic, Nonlinear and Adaptive Control Design, New York: Wiley (1995)

\section{《付録》}

\section{A. 出カフィードバック指数受動性の証明}

OFEP 条件を満足する標準形に対し, 入力 (3.3) を用い棈 成された閉ループ系は

$$
\begin{aligned}
& \dot{y}(t)=\widetilde{a}\left(y, z_{q}\right)-\widetilde{B}_{0} K \boldsymbol{y}(t)+\widetilde{B}_{0} v(t) \\
& \dot{z}_{q}(t)=\boldsymbol{q}\left(\boldsymbol{y}, \boldsymbol{z}_{q}\right)
\end{aligned}
$$

となる。OFEP 条件 (2),(3) 上り，(3.12) を满足する正定値関 数 $W\left(\boldsymbol{z}_{q}\right)$ が存在する. そこで，つぎの正定值関数を考える.

$$
V_{1}\left(\boldsymbol{y}, z_{q}\right)=W\left(z_{q}\right)+\frac{1}{2} \boldsymbol{y}(t)^{T} \widetilde{B}_{0}^{-1} \boldsymbol{y}(t)
$$

$V_{1}\left(\boldsymbol{y}, z_{q}\right)$ の時間微分は,

$$
\begin{aligned}
\dot{V}_{1}= & \frac{\partial W\left(z_{q}\right)}{z_{q}} \boldsymbol{q}\left(\boldsymbol{y}, \boldsymbol{z}_{q}\right)+\boldsymbol{y}^{T} \widetilde{B}_{0}^{-1} \widetilde{a}\left(\boldsymbol{y}, z_{q}\right) \\
& -\frac{1}{2} \boldsymbol{y}^{T}\left(K^{T}+K\right) \boldsymbol{y}+\boldsymbol{y}^{T} \boldsymbol{v}
\end{aligned}
$$

となる.ここで, OFEP 条件 (3) および (3.12)より, $\boldsymbol{q}\left(\boldsymbol{y}, \boldsymbol{z}_{q}\right)$ および $\widetilde{a}\left(\boldsymbol{y}, \boldsymbol{z}_{q}\right)$ に対する Lipschitz 定数をそれぞれ $L_{1}, L_{2}$ と おくと

$$
\begin{aligned}
& \frac{\partial W\left(\boldsymbol{z}_{q}\right)}{\boldsymbol{z}_{q}} \boldsymbol{q}\left(\boldsymbol{y}, \boldsymbol{z}_{q}\right) \leq-\alpha_{1}\left\|\boldsymbol{z}_{q}\right\|^{2}+\alpha_{2} L_{1}\left\|z_{q}\right\|\|\boldsymbol{y}\|(\mathrm{A} .4) \\
& \boldsymbol{y}^{T} \widetilde{B}_{0}^{-1} \widetilde{\boldsymbol{a}}\left(\boldsymbol{y}, \boldsymbol{z}_{q}^{\prime}\right) \leq\left\|\widetilde{B}_{0}^{-1}\right\| L_{2}\left(\|\boldsymbol{y}\|\left\|\boldsymbol{z}_{q}\right\|+\|\boldsymbol{y}\|^{2}\right)(\mathrm{A} .5)
\end{aligned}
$$

と評価できることから， $\dot{V}_{1}$ は，

$$
\begin{aligned}
\dot{V}_{1} \leq & -\alpha_{1}\left\|\boldsymbol{z}_{q}\right\|^{2}+\left(\alpha_{2} L_{1}+\left\|\widetilde{B}_{0}^{-1}\right\| L_{2}\right)\left\|\boldsymbol{z}_{q}\right\|\|\boldsymbol{y}\| \\
& -\frac{1}{2} \lambda_{\min }\left(K^{T}+K\right)\|\boldsymbol{y}\|^{2}+\left\|\widetilde{B}_{0}^{-1}\right\| L_{2}\|\boldsymbol{y}\|^{2}+\boldsymbol{y}^{T} \boldsymbol{v} \\
\leq & -\left(\alpha_{1}-\rho_{0}\right)\left\|\boldsymbol{z}_{q}\right\|-\left(\frac{1}{2} \lambda_{\min }\left(K^{T}+K\right)-\beta_{0}\right)\|\boldsymbol{y}\|^{2} \\
& +\boldsymbol{y}^{T} \boldsymbol{v}
\end{aligned}
$$

と評価できる。ここに， $\rho_{0}$ は, 任意の正定数であり， $\beta_{0}$ は,

$$
\beta_{0}=\left\|\widetilde{B}_{0}^{-1}\right\| L_{2}+\frac{\left(\alpha_{2} L_{1}+\left\|\widetilde{B}_{0}^{-1}\right\| L_{2}\right)^{2}}{4 \rho_{0}}
$$

である。このとき， $0<\rho_{0}<\alpha_{1}$ ととると

$$
\lambda_{\min }\left(K^{T}+K\right)>\beta_{0}
$$

なるすべての $K に$ 対し， $\dot{V}_{1}$ は,

$$
\dot{V}_{1} \leq-c_{1}\left\|\boldsymbol{z}_{q}\right\|^{2}-c_{2}\|\boldsymbol{y}\|^{2}+\boldsymbol{y}^{T} \boldsymbol{v}, c_{1}, c_{2}>0
$$

と評価できる。結局, $\lambda_{\min }\left(K_{0}\right)=\beta_{0}$ なる $K_{0}=K_{0}^{T}>0$ に 対し， $K^{T}+K>K_{0}$ なるすべての $K$ で構成された閉ループ 系はvからyに関して指数受動となることがわかる。

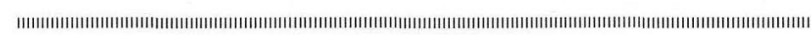

$$
\text { [著 者 紹 介] }
$$

\section{水 本 郁 朗 (正会員)}

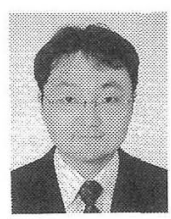

1991 年熊本大学大学院工学研究科修士課程 (機 械工学専攻) 修了. 同年同大学工学部機械工学科 助手, 97 年同大学工学部知能生産システム工学科 助教授, 現在, 同大学大学院自然科学研究科助教 授. 適応制御などの制御理論に関する研究に従事. 日本機械学会, システム制御情報学会などの会員 (博士 (工学) ).

\section{岩 井善 太（正会員）}

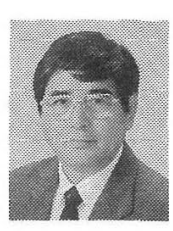

1964 年京都大学工学部数理工学科卒業, 66 年 京都大学大学院工学研究科修士課程 (数理工学専 攻) 修了. 現在, 熊本大学工学部知能生産システ ム工学科教授。適応制御などの制御理論およびそ の応用に関する研究に従事. 日本機械学会, シス テム制御情報学会などの会員（工学博士）.

小 原 浩司朗

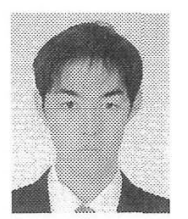

1999 年熊本大学工学部機械工学科卒業. 同年 同大学大学院自然科学研究科博士前期課程 (機械 システム専攻) 入学, 現在に至る. 非線形適応制 御の研究に従事.

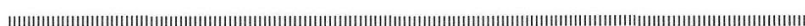

